\title{
The approval of a disease-modifying treatment for Alzheimer's disease: impact and consequences for the nuclear medicine community
}

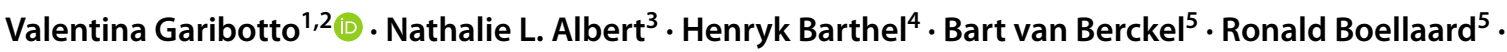 \\ Matthias Brendel ${ }^{3,6,7} \cdot$ Diego Cecchin $^{8} \cdot$ Ozgul Ekmekcioglu $^{9} \cdot$ Elsmarieke van de Giessen $^{5} \cdot$ Eric Guedj $^{10}$. \\ Adriaan A. Lammerstma ${ }^{5,11}$. Franck Semah ${ }^{12}$. Tatjana Traub-Weidinger ${ }^{13}$. Donatienne Van Weehaeghe ${ }^{14}$. \\ Silvia Morbelli ${ }^{15}$. for the EANM Neuroimaging Committee
}

Published online: 17 July 2021

(C) The Author(s), under exclusive licence to Springer-Verlag GmbH Germany, part of Springer Nature 2021

On June 7th, 2021, for the first time since 2003, a novel drug against Alzheimer's disease (AD), aducanumab (Aduhelm ${ }^{\mathrm{TM}}$ ), has been approved in the USA by the Food and Drug Administration (FDA).

This event already has had a major impact, as shown by the storm of commentaries, both in favor and against it, in journals and social media before and after its disclosure. Its impact is linked to a number of factors: AD is a devastating and incurable disease with a dramatic toll on both patients and their families; Aducanumab is the first approved drug that was tested for its ability to influence the course of the disease (disease-modifying drug) and not only to treat

This article is part of the Topical Collection on Neurology

Valentina Garibotto

valentina.garibotto@hcuge.ch

1 Nuclear Medicine and Molecular Division, Diagnostic Department, University Hospitals of Geneva, Rue Gabrielle-Perret-Gentil 4, 1205 Geneva, Switzerland

2 NIMTLab, Faculty of Medicine, University of Geneva, Geneva, Switzerland

3 Department of Nuclear Medicine, University Hospital of Munich, LMU Munich, Munich, Germany

4 Department of Nuclear Medicine, University Hospital Leipzig, Leipzig, Germany

5 Department of Radiology and Nuclear Medicine, Amsterdam University Medical Centers, Location VUMC, Amsterdam, the Netherlands

6 DZNE - German Center for Neurodegenerative Diseases, Munich, Germany

7 SyNergy, University of Munich, Munich, Germany

8 Nuclear Medicine Unit, Department of Medicine (DIMED), University-Hospital of Padua, Padua, Italy symptoms; the FDA decision was taken on the basis of two phase III trials with discordant results on clinical efficacy, which first were interrupted on the basis of a futility analysis, but subsequently revived (for a detailed summary, see [1]); the FDA decision was strongly based on the amyloidclearing capacity of the drug, as shown by amyloid PET imaging.

The scientific and clinical community is cleaved in two fields: on the one hand, those who welcome this decision as a step in the right direction, finally offering a therapeutic option to patients and fostering research and development in this direction, and on the other hand, those considering this decision as driven mainly by expectations and lacking solid scientific evidence.

$9 \quad$ Nuclear Medicine Department, Sisli Hamidiye Etfal Education and Research Hospital, University of Health Sciences, Istanbul, Turkey

10 Nuclear Medicine Department, Institut Fresnel, APHM, CNRS, Timone Hospital, CERIMED, Aix Marseille Univ, Centrale Marseille, Marseille, France

11 Medical Imaging Center, Department of Nuclear Medicine and Molecular Imaging, University of Groningen, University Medical Center Groningen, Groningen, The Netherlands

12 Department of Nuclear Medicine, Univ Lille, CHU, Lille, France

13 Division of Nuclear Medicine, Department of Biomedical Imaging and Image-Guided Therapy, Medical University of Vienna, Vienna, Austria

14 Nuclear Medicine, UZ Leuven, Leuven, Belgium

15 Nuclear Medicine Unit, IRCCS Ospedale Policlinico San Martino, Genoa, Italy 
The aim of this commentary is not to take one or the other position, but rather to highlight the impact and consequences of this development for the nuclear medicine community.

The practical impact of this decision is still difficult to appreciate: the FDA approval was obtained under the accelerated approval pathway, which requires evidence to be collected in a post-marketing trial that will have to be launched. The drug is also under evaluation by the European Medicinal Agency (EMA) and other national agencies and the debate will surely continue over the next months. Specific criteria for eligibility and monitoring will have to be defined and access to imaging and drug negotiated on a national basis.

We will focus here on two main aspects directly concerning our clinical and research practice: (1) using amyloid PET imaging as eligibility criterion and anticipating the associated needs, and (2) evaluating amyloid PET readout changes as surrogate outcome measures in clinical trials.

The use of amyloid PET to select patients eligible for the treatment will be based on the well-characterized diagnostic performance of this approach [2]. Amyloid imaging has been extensively used in therapeutic trials of amyloid-targeting agents as inclusion criterion/sample enrichment tool. Its use was motivated by the evidence that trials solely based on clinical criteria had a high proportion (as high as 30\%) of amyloid-negative subjects who, as such, did not possess the actual drug target [3].

The phase III aducanumab trials indeed included screening with amyloid PET as proof of amyloid positivity for subjects to be eligible for the drug. Consequently, it is expected that this criterion will be maintained when the drug will be used clinically. This will surely represent an increase in amyloid PET prescription. While amyloid positivity can also be indirectly assessed by the dosage of amyloid and tau in the CSF, patients with contraindications or refusing the procedure, as well as in patients in which CSF results are inconclusive, represent a non-negligible proportion, up to $30-35 \%$ of patients [4-6]. Furthermore, the added incremental diagnostic value of amyloid PET over CSF has been demonstrated [7-9]. Finally, blood markers for amyloid and tau have seen a very promising development over the last years and have already fulfilled the first steps in view of a clinical validation. Thus, we might expect soon their introduction into clinical practice [10]. However, even when they will be available, it is most likely that a two-step procedure will be adopted, with a blood-based screening followed by a confirmation with a more accurate diagnostic strategy, presumably imaging, in an integrated diagnostic approach [11]. Finally, as the approval of the drug is based on the ability of the drug to reduce amyloid deposits, it might be expected that imaging, the only method allowing a measurement of brain amyloid load, will have a role in treatment monitoring. The costs of PET imaging are lower that the projected costs of the treatment. Thus, the use of PET as intermediate assessment, if proven, could even be economically advantageous.

On the basis of these elements, the nuclear medicine community, including tracer providers and imaging sites, projects an increase in the need of the amyloid PET as high as 20-fold and will have to guarantee a timely access to the technology and to ensure proper training of a large number of nuclear medicine physicians.

The use of amyloid PET imaging as surrogate outcome measure of the effect of anti-AD drugs is a more complex matter and has been less explored. The main motivations for using a biomarker, such as PET imaging, as surrogate marker in therapeutic trials are as follows:

- the differentiation between disease-modifying and symptomatic effects. While symptomatic effects remain the primary outcome measure of clinical trials, the assessment of disease-modifying effects in a slowly progressive process, such as neurodegeneration, is difficult to target solely on the basis of cognitive measures. Specific trial designs exist to address this question, such as the "delayed-start" design, but biomarkers, namely molecular imaging biomarkers, have the advantage to show actual changes in pathology non-invasively.

- the quantitative nature of the test. Clinical/cognitive scales have a higher test/retest variability. Using more direct and quantitative measures of drug action could allow for increasing statistical power, thereby reducing sample sizes, with major economic advantages: indeed, despite the fact that PET imaging is per se a relatively expensive procedure, its costs are low as compared with the costs associated with the clinical trial of an experimental drug.

A specific regulatory framework exists, summarizing the main requirements for the use of a biomarker for treatment monitoring and evaluation of outcome, focusing mainly on the association between biomarker and clinical outcome and between biomarker and pathophysiology [12]. As a treatment biomarker, amyloid PET clearly fulfills the criterion of being associated with the pathological phenomenon targeted by the therapy and of being linked to its disease-modifying potential. This is while no direct correlation with the clinical outcome, which is the main outcome of the majority of the trials, is necessarily expected in the clinical disease phase, as-in the current view-pathological amyloid is not the major driver of symptoms.

Both EMA and FDA have recently published recommendations for the use of biomarkers in clinical trials of treatments targeting $\mathrm{AD}$, setting the framework for the role that PET molecular imaging could play in this context $[13,14]$.

Both recommendations recognize a shift of the clinical trial design, rational, and strategies in $\mathrm{AD}$ in order to target 
the earliest symptomatic or even preclinical stages. They also consider the major changes over the last decade in the diagnostic criteria adopted for the disease, providing an increasing place to the use of biomarkers $[15,16]$

The FDA guidance draft, distributed in February 2018 for comments, opened the way to consider pathophysiological AD imaging markers as surrogate markers in two main cases: (1) in patients with "subtle detectable abnormalities on sensitive neuropsychological measures," defined as stage 2 , for whom imaging could be a supportive marker for marketing approval; (2) in stage 1 subjects, i.e., subjects with pathophysiologic changes of AD but no evidence of clinical impact, for whom biomarker changes could be the primary efficacy measure of the trial.

The EMA guideline supports the use of biomarkers in the different phases of drug development, namely for early pharmacological and pharmacokinetic studies and exploratory trials, and their potential role as prognostic and predictive markers, or pharmacodynamics markers, i.e., for determination of biological effects of the drug. The EMA guideline however does not consider the use of biomarkers as outcome measures, not even in studies focusing on presymptomatic cases.

Amyloid PET has been evaluated in a number of recent clinical anti-amyloid drug testing trials as secondary outcome measure. When considering published results, while many drugs showed no or only a slight reduction in amyloid load, as, e.g., bapineuzumab [17], solanezumab [18], or crenezumab [19], some more recently developed molecules have shown larger effects on amyloid load including aducanumab [20], donanemab [21], gantenerumab [22], and lecanemab [23].

These changes have been measured as changes in standardized uptake value ratios (SUVr) between a set of cortical regions and a reference region, often the cerebellum (or white matter), on static images acquired at fixed time points after injection. It is important to emphasize that a specific validation process is required when a PET tracer is introduced in a clinical trial as treatment biomarker, even when this tracer is well known and characterized for its diagnostic properties. Indeed, the effects of the investigational drug on the tracer's biodistribution, namely on brain perfusion/whole body excretion and first pass extraction (blood-brain-barrier effects), should be accurately measured and taken into account when selecting the outcome parameter. SUVr is a biased measure for assessment of reduction of amyloid load after a pharmacotherapeutic intervention, as it shows a "reduction" in amyloid load over time in $\mathrm{AD}$ patients that in fact is an artefact linked with the perfusion reduction in cortical areas that occurs in $\mathrm{AD}$ patients over time $[24,25]$. This effect could be canceled out in a randomized trial if it is comparable in both active and placebo arms, but only if the experimental drug has no specific effect on perfusion and this requires specific validation. The added value of dynamic
PET imaging in clinical trials to address these aspects has been explored previously [26, 27]. SUVr-independent methods and grading of amyloid PET results incorporating multiple metrics have been already tested in multi-center and multi-tracer studies [28]. As a viable compromise between longer scan times required for dynamic imaging and patient comfort, dual-time window protocols guarantying full-quality dynamic amyloid load readouts have recently been proposed $[29,30]$.

PET biomarkers are one of the key tools in the hands of the scientific community in the quest for the long-awaited effective therapies for AD. With the first disease-modifying drug for $\mathrm{AD}$ entering clinical practice, it is more important than ever to ensure that molecular imaging is ready to support patient evaluation. This requires a rigorous a priori validation to optimize the information that can be provided.

\section{Declarations}

Ethics approval Institutional Review Board approval was not required because the paper is an Editorial.

Informed consent Not applicable.

Consent to participate Not applicable.

Conflict of interest Valentina Garibotto reports grants from the Swiss National Science Foundation (projects 320030_169876, 320030_185028 and IZSEZ0_188355) the Schmidheiny foundation and the Velux foundation (project 1123). Valentina Garibotto received financial support for research and/or speaker fees through her institution from Siemens Healthineers, GE Healthcare, Life Molecular Imaging, Cerveau Technologies, Roche, Merck.

Bart van Berckel received research support from ZON-MW, AVID radiopharmaceuticals, CTMM, and Janssen Pharmaceuticals. He is a trainer for Piramal and GE. He receives no personal honoraria.

Henryk Barthel received speaker honoraria from Novartis/AAA. Matthias Brendel reports grants by the German Research Foundation (projects BR4580/1-1; EXC 2145 SyNergy - ID 390857198), LMU Munich (project 980), and the Alzheimer Forschung Initiative e.V. (project 19063p). Matthias Brendel received speaker honoraria from GE healthcare, Roche, and Life Molecular Imaging, and is an advisor of Life Molecular Imaging.

Diego Cecchin received free doses for CE approved amyloid PET acquisitions form Life Molecular Imaging and from GE Healthcare in 2019-2021.

Eric Guedj received financial support from Life Molecular Imaging for an amyloid PET project in 2017; and from GE Healthcare, Curium, and AAA/Novartis on topics unrelated to this article.

Silvia Morbelli received speaker honoraria from GE healthcare.

All other authors have no conflicts of interest to disclose.

\section{References}

1. Kuller LH, Lopez OL. ENGAGE and EMERGE: truth and consequences? Alzheimers Dement 2021;17:692-5. https://doi.org/ 10.1002/alz.12286. 
2. Chetelat G, Arbizu J, Barthel H, Garibotto V, Law I, Morbelli S, et al. Amyloid-PET and (18)F-FDG-PET in the diagnostic investigation of Alzheimer's disease and other dementias. Lancet Neurol 2020;19:951-62. S1474-4422(20)30314-8 [pii] https://doi.org/ 10.1016/S1474-4422(20)30314-8.

3. Barthel H, Seibyl J, Sabri O. The role of positron emission tomography imaging in understanding Alzheimer's disease. Expert Rev Neurother 2015;15:395-406. https://doi.org/10.1586/14737175. 2015.1023296.

4. Duits FH, Prins ND, Lemstra AW, Pijnenburg YA, Bouwman $\mathrm{FH}$, Teunissen CE, et al. Diagnostic impact of CSF biomarkers for Alzheimer's disease in a tertiary memory clinic. Alzheimers Dement 2015;11:523-32. https://doi.org/10.1016/j.jalz.2014.05. 1753.

5. Herukka SK, Simonsen AH, Andreasen N, Baldeiras I, Bjerke $\mathrm{M}$, Blennow K, et al. Recommendations for cerebrospinal fluid Alzheimer's disease biomarkers in the diagnostic evaluation of mild cognitive impairment. Alzheimers Dement 2017;13:285-95. https://doi.org/10.1016/j.jalz.2016.09.009.

6. Nath S, Badhiwala JH, Alhazzani W, Nassiri F, Belley-Cote E, Koziarz A, et al. Atraumatic versus traumatic lumbar puncture needles: a systematic review and meta-analysis protocol. BMJ Open 2017;7:e014478. https://doi.org/10.1136/bmjop en-2016-014478.

7. Ceccaldi M, Jonveaux T, Verger A, Krolak-Salmon P, Houzard C, Godefroy O, et al. Added value of (18)F-florbetaben amyloid PET in the diagnostic workup of most complex patients with dementia in France: a naturalistic study. Alzheimers Dement 2018;14:293305. https://doi.org/10.1016/j.jalz.2017.09.009.

8. Ramusino MC, Garibotto V, Bacchin R, Altomare D, Dodich A, Assal F, et al. Incremental value of amyloid-PET versus CSF in the diagnosis of Alzheimer's disease. Eur J Nucl Med Mol Imaging 2020;47:270-80. https://doi.org/10.1007/s00259-019-04466 [pii].

9. Reimand J, Collij L, Scheltens P, Bouwman F, Ossenkoppele R. Association of amyloid-beta CSF/PET discordance and tau load 5 years later. Neurology 2020;95:e2648-57. https://doi.org/10.1212/ WNL.0000000000010739WNL.0000000000010739[pii].

10. Ashton NJ, Leuzy A, Karikari TK, Mattsson-Carlgren N, Dodich A, Boccardi M, et al. The validation status of blood biomarkers of amyloid and phospho-tau assessed with the 5-phase development framework for AD biomarkers. Eur J Nucl Med Mol Imaging 2021;48:2140-56. https://doi.org/10.1007/s00259-021-05253-y.

11. Garibotto V, Boccardi M, Chiti A, Frisoni GB. Molecular imaging and fluid biomarkers of Alzheimer's disease neuropathology: an opportunity for integrated diagnostics. Eur J Nucl Med Mol Imaging 2021;48:2067-9. https://doi.org/10.1007/ s00259-020-05116-y.

12. Broich K, Weiergraber M, Hampel H. Biomarkers in clinical trials for neurodegenerative diseases: regulatory perspectives and requirements. Prog Neurobiol 2011;95:498-500.

13. EMA. Guideline on the clinical investigation of medicines for the treatment of Alzheimer's disease. 2018.

14. FDA. Early Alzheimer's disease: developing drugs for treatment. Guidance for Industry. 2018.

15. Jack CR Jr, Bennett DA, Blennow K, Carrillo MC, Dunn B, Haeberlein SB, et al. NIA-AA Research Framework: toward a biological definition of Alzheimer's disease. Alzheimers Dement 2018;14:535-62. https://doi.org/10.1016/j.jalz.2018.02.018.

16. Dubois B, Feldman HH, Jacova C, Hampel H, Molinuevo JL, Blennow K, et al. Advancing research diagnostic criteria for Alzheimer's disease: the IWG-2 criteria. Lancet Neurol 2014;13:614 29. https://doi.org/10.1016/S1474-4422(14)70090-0.

17. Salloway S, Sperling R, Fox NC, Blennow K, Klunk W, Raskind $\mathrm{M}$, et al. Two phase 3 trials of bapineuzumab in mild-to-moderate Alzheimer's disease. N Engl J Med 2014;370:322-33. https://doi. org/10.1056/NEJMoa1304839.
18. Honig LS, Vellas B, Woodward M, Boada M, Bullock R, Borrie M, et al. Trial of solanezumab for mild dementia due to Alzheimer's disease. N Engl J Med 2018;378:321-30. https://doi.org/10. 1056/NEJMoa1705971.

19. Salloway S, Honigberg LA, Cho W, Ward M, Friesenhahn M, Brunstein F, et al. Amyloid positron emission tomography and cerebrospinal fluid results from a crenezumab anti-amyloid-beta antibody double-blind, placebo-controlled, randomized phase II study in mild-to-moderate Alzheimer's disease (BLAZE). Alzheimers Res Ther 2018;10:96. https://doi.org/10.1186/ s13195-018-0424-5pii].

20. Sevigny J, Chiao P, Bussiere T, Weinreb PH, Williams L, Maier $\mathrm{M}$, et al. The antibody aducanumab reduces Abeta plaques in Alzheimer's disease. Nature 2016;537:50-6. https://doi.org/10.1038/ nature19323nature19323[pii].

21. Mintun MA, Lo AC, Duggan Evans C, Wessels AM, Ardayfio PA, Andersen SW, et al. Donanemab in early Alzheimer's disease. $\mathrm{N}$ Engl J Med 2021. https://doi.org/10.1056/NEJMoa2100708

22. Klein G, Delmar P, Kerchner GA, Hofmann C, Abi-Saab D, Davis A, et al. Thirty-six-month amyloid positron emission tomography results show continued reduction in amyloid burden with subcutaneous gantenerumab. J Prev Alzheimers Dis 2021;8:3-6. https:// doi.org/10.14283/jpad.2020.68.

23. Swanson CJ, Zhang Y, Dhadda S, Wang J, Kaplow J, Lai RYK, et al. A randomized, double-blind, phase $2 b$ proof-of-concept clinical trial in early Alzheimer's disease with lecanemab, an anti-Abeta protofibril antibody. Alzheimers Res Ther 2021;13:80. https://doi.org/10.1186/s13195-021-00813-8[pii].

24. van Berckel BN, Ossenkoppele R, Tolboom N, Yaqub M, FosterDingley JC, Windhorst AD, et al. Longitudinal amyloid imaging using 11C-PiB: methodologic considerations. J Nucl Med 2013;54:1570-6. https://doi.org/10.2967/jnumed.112.11365 4jnumed.112.113654[pii].

25. Barthel H, Seibyl J, Lammertsma AA, Villemagne VL, Sabri O. Exploiting the full potential of beta-amyloid and tau PET imaging for drug efficacy testing. J Nucl Med 2020;61:1105-6. https://doi. org/10.2967/jnumed.119.228346.

26. Ossenkoppele R, Prins ND, van Berckel BN. Amyloid imaging in clinical trials. Alzheimers Res Ther 2013;5:36. https://doi.org/10. 1186/alzrt195.

27. Lopes Alves I, Heeman F, Collij LE, Salvado G, Tolboom N, Vilor-Tejedor N, et al. Strategies to reduce sample sizes in Alzheimer's disease primary and secondary prevention trials using longitudinal amyloid PET imaging. Alzheimers Res Ther 2021;13:82. https://doi.org/10.1186/s13195-021-00819-2[pii].

28. Chincarini A, Peira E, Morbelli S, Pardini M, Bauckneht M, Arbizu J, et al. Semi-quantification and grading of amyloid PET: a project of the European Alzheimer's Disease Consortium (EADC). Neuroimage Clin 2019;23:101846. https://doi.org/10. 1016/j.nicl.2019.101846.

29. Bullich S, Barthel H, Koglin N, Becker GA, De Santi S, Jovalekic A, et al. Validation of noninvasive tracer kinetic analysis of (18) F-florbetaben PET using a dual-time-window acquisition protocol. J Nucl Med 2018;59:1104-10. https://doi.org/10.2967/jnumed. 117.200964.

30. Heeman F, Yaqub M, Lopes Alves I, Heurling K, Berkhof J, Gispert JD, et al. Optimized dual-time-window protocols for quantitative [(18)F]flutemetamol and [(18)F]florbetaben PET studies. EJNMMI Res 2019;9:32. https://doi.org/10.1186/ s13550-019-0499-4.

Publisher's note Springer Nature remains neutral with regard to jurisdictional claims in published maps and institutional affiliations. 